\title{
EDITORIAL
}

\section{Microbial ecologists: it's time to 'go large'}

\author{
The extent of microbial diversity in the environment is beginning to be assessed by \\ environmental genomics studies, but are these studies large enough? Here, Tom Curtis \\ issues a call to arms for microbial ecologists - it's time to start thinking big.
}

As the post-Pace revolution continues, new methods in microbial ecology are being invented and deployed, and new wonders seen in both noble (the seas and soil) and prosaic (the intestines and sewage works) environments. It is therefore a good time to reflect on the magnitude of the task that microbial ecologists face in their efforts to analyse the microbial majority.

What are the facts? Microorganisms are small, they are extremely important and there is an awful lot of them $-10^{30}$ to be inexact. But we have only a vague idea of the extent of microbial diversity, with estimates differing by factors of 1,000 or more. We have even less of an idea about the proportional abundance of microorganisms, and not much of a clue how these patterns do and do not change. This is not simply a search for trivial facts. These concepts underlie all explorations in, and exploitations of, the microbial world.

So why are we still in the dark? There are many considerations, but the single factor that outweighs all others is sample size - we are looking at vast systems with pathetically small samples. A clone library of 1,000 might sound fine but in a community of $10^{18}$ it is modest: one clone for every $10^{12}$ individuals. Today, sample sizes are dictated by budgets and not by a rational consideration of what is required. This is not a comfortable thought. Sample sizes of tens or even hundreds of thousands might be required to adequately sample diversity, yet these numbers are beyond the current modus operandi of most laboratories.

Nevertheless, this is an issue that we need to tackle head on. After all, contemporary microbial ecology studies are not cheap; we are not saving money by doing the job inadequately. Moreover, once we commit to doing a proper job, the job itself will become easier. Simply acknowledging the size of the problem and giving kudos to those who contribute to its solution will yield faster, better and cheaper methods. More subtly, models to predict diversity - validated with large samples but calibrated using small ones - could be used to approximately describe environments that we cannot yet afford to explore.

Ultimately, we must face up to the scale of the microbial world, retool and 'go large'. To escape from the Captain Cook phase of one-off heroic explorations, we must industrialize and automate sample processing and analysis. The task should be given over to microbial equivalents of the US or British Geological Surveys. A 'Microbial Survey' could run, or subcontract, sequencing factories for the community at large, and explore the microbial world systematically itself using a judicious mix of deep-sequencing and mathematical modelling.

There has been pioneering work, such as the US National Science Foundation (NSF) Microbial Observatories programme and the UK National Environmental Research Council (NERC) thematic studies of soil and marine microbial diversity, as well as the Genomes to Life programme of the US Department of Energy's Joint Genome Institute (JGI). But the NSF and NERC programmes are either explicitly (in the UK) or implicitly (in the US) of finite tenure as microorganisms must take their place with other ecology programmes, and the 'factories' we have in the JGI and the Wellcome Trust Sanger Institute must target the needs of their sponsors in energy and medicine. However, the challenge of diversity is not confined to a single domain of science and is certainly not a burden that microbial ecology, medicine or energy budgets should bear alone. Engineering and agriculture, medicine and veterinary science, meteorology and even space flight are all profoundly affected by microorganisms.

This will cost, of course. But we have a model in the British Geological Survey and its US counterpart. Working with just $10 \%$ of the budgets of these organizations, ( $£ 5$ million and $\$ 93$ million, respectively) we could revolutionize the exploration of the microbial world. As I look ahead, it is easy to see that microorganisms could be just as important to our health, wealth and well-being in the twenty-first century as geology and geomatics were in the nineteenth and twentieth centuries.

I make no apologies for putting microorganisms on a pedestal above all other living things. For if the last blue whale choked to death on the last panda, it would be disastrous but not the end of the world. But if we accidentally poisoned the last two species of ammonia-oxidizers, that would be another matter. It could be happening now and we wouldn't even know... 